\title{
TERRITÓRIO ALIMENTAR EM DISPUTA: A CONSTITUIÇÃO DE DESERTOS E PÂNTANOS ALIMENTARES A PARTIR DA LÓGICA DE DISTRIBUIÇÃO DE ULTRAPROCESSADOS
}

\section{FOOD TERRITORY IN DISPUTE: THE CONSTITUTION OF FOOD DESERTS AND FOOD SWAMPS FROM THE LOGIC OF ULTRAPROCESSED DISTRIBUTION}

\author{
Mateus Luciani dos Santos \\ Universidade Federal do Paraná, Departamento de Geografia, Curitiba, PR, Brasil \\ mateusluciani@gmail.com \\ Pedro Augusto Breda Fontão \\ Universidade Federal do Paraná, Departamento de Geografia, Curitiba, PR, Brasil \\ pedrofontao@ufpr.br
}

\begin{abstract}
RESUMO
O objetivo deste artigo é discutir as dualidades entre o sistema alimentar moderno, vinculado à produção de alimentos ultraprocessados por grandes corporações ligadas à alimentação, em oposição ao modelo tradicional, vinculado a produtos in natura com qualidade nutricional. Busca-se compreender as políticas do estado brasileiro quanto à garantia do direito humano à alimentação saudável (DHAA). Metodologicamente o artigo é construído a partir de uma revisão sistemática da literatura, utilizando bases Miltonianas como a de Saquet e Silva (2008) e Abrão (2010) para a compreensão do problema proposto. O resultado encontrado pelo estudo é que a capacidade de articulação da grande indústria alimentícia promove um território alimentar que está em uma disputa perene, especialmente devido ao amplo poder da grande indústria de se perpetuar pelo território inserindo seus produtos. Conclui-se portanto que o estado brasileiro dispõe de um arcabouço legislativo com intuito de garantir o DHAA. Entretanto, por fatores vinculados ao ambiente político e ao próprio interesse dos grandes grupos alimentares, as políticas públicas não são colocadas em prática de sua forma completa. O combate a esses problemas deve ser pautado politicamente, dessa forma podemos utilizar a dinâmica territorial do Sistema Único de Saúde (SUS) para solucionar esse problema.
\end{abstract}

Palavras-chave: Direito Humano à Alimentação Saudável (DHAA). Desertos e pântanos alimentares. Política Nacional de Alimentação. Território e saúde. Saúde alimentar.

\begin{abstract}
The objective of this paper is to discuss the dualities between the modern food system, linked to the production of ultra-processed foods by large food corporations, as opposed to the traditional model, linked to fresh products with nutritional quality. It seeks to understand the policies of the Brazilian state regarding the guarantee of the human right to healthy food (DHAA). Methodologically, the article is built from a systematic literature review, using Miltonian bases such as Saquet and Silva (2008) and Abrão (2010) to understand the proposed problem. The result found by the study is that the articulation capacity of the large food industry promotes a food territory that is in a permanent dispute, especially due to the large industry's broad power to perpetuate itself through the territory by inserting its products. The conclusion is that the Brazilian state has a legislative framework in place in order to guarantee the DHAA. However, due to factors related to the political environment and the interests of the large food groups, public policies are not fully implemented. The action against these problems must be politically guided, so we can use the territorial dynamics of the Unified Health System (SUS) to solve this problem.
\end{abstract}

Keywords: Human Right to Healthy Eating (HRHE). Food deserts and food swamps. National Food Policy. Territory and health. Food health

Recebido em: 03/01/2022

Aceito para publicação em: 27/01/2022.

Hygeia Uberlândia - MG $\quad$ Edição especial: X GeoSaude $\quad$ Fev./2022 $\quad$ p. 34-45 Página 34




\section{INTRODUÇÃO}

A alimentação é um direito básico e necessário para a sobrevivência dos seres humanos, dado que se alimentar diariamente e de forma equilibrada é algo essencial para garantir a saúde e a qualidade de vida dos indivíduos. Em função disso, Fome Zero e Agricultura Sustentável é o segundo item dos 17 Objetivos de Desenvolvimento Sustentável (ODS) da Agenda 2030, proposta e adotada pela Organização das Nações Unidas (ONU), cujo foco é acabar com a fome, alcançar a segurança alimentar e melhoria da nutrição e promover a agricultura sustentável.

Nesse aspecto, é fundamental garantir o acesso de todas as pessoas a alimentos seguros e nutritivos, em particular as populações mais pobres e pessoas em situações vulneráveis. No entanto, num mundo cada vez mais globalizado, em que existe o predomínio de grandes grupos econômicos orientando o nosso cotidiano em uma gama de atividades, o setor alimentar também encontra-se sujeito a este cenário. Por conseguinte, nas últimas décadas a inserção e expansão dos grandes conglomerados alimentares trouxe uma série de implicações sobre o modo que as pessoas satisfazem suas necessidades calóricas diárias, sendo que uma delas pode ser verificada na substituição de alimentos in natura, com o mínimo processamento, por produtos e alimentos denominados ultraprocessados, em que basicamente tratam-se de "formulações industriais à base de ingredientes extraídos ou derivados de alimentos (óleos, gorduras, açúcar, amido modificado) ou, ainda, sintetizados em laboratório (corantes, aromatizantes, realçadores de sabor, etc.)" (BRASIL, 2014, p. 8).

No tocante aos alimentos ultraprocessados, seus insumos são obtidos a partir de reconstituições de componentes alimentares de uma gama de commodities agroindustriais como milho, soja, leite, açúcar, trigo e óleo de palma, tendo como resultado uma formulação alimentar de baixa qualidade (SCRINIS, 2016). Monteiro et al. (2010) e Martins et al. (2013) ponderam que essa classe de alimentos apresentam características próprias, onde o alimento em si apresenta maior densidade calórica e uma menor quantidade proteínas e fibras em comparação com alimentos in natura. Por causa dessas peculiaridades, esses alimentos favorecem o consumo excessivo e desmedido, de modo que um produto ultraprocessado apresenta valores de gorduras saturadas, totais, açúcares e sódio em concentrações acima dos padrões nutricionais recomendados.

Desse modo, este artigo tem o intuito de dialogar e realizar uma breve avaliação das cadeias de distribuição alimentares sobre o território e o seu impacto na produção de zonas de desertos alimentares e pântanos alimentares, além de buscar compreender a lógica das políticas de Estado como políticas de saúde pública pensadas sob a ótica territorial, e como elas podem (ou poderiam) evitar a formação dessas áreas de alimentação precária e contribuir para a melhoria da saúde e das condições de vida da população. Além disso, o artigo buscará compreender também como a crise alimentar oriundo da pandemia de COVID-19 impactou na alimentação da população brasileira, em especial dos mais pobres.

\section{PROCEDIMENTOS METODOLÓGICOS}

Para amparar e suscitar esta investigação, foi necessário, inicialmente, realizar um levantamento e observação dos principais conceitos e concepções que envolvem essa temática em destaque. Em primeiro lugar, foi feita uma pesquisa e revisão bibliográfica sobre o uso do conceito de ultraprocessados e suas respectivas cadeias de distribuição, bem como o congruente processo de formação de desertos e pântanos alimentares, dando ênfase para alguns estudos recentes e a abordagem de autores como Shaw (2006), Vandevijvere et al. (2019), Honório (2020), entre outros. A partir de um ponto de vista dialético, avaliou-se o contexto recente dos ultraprocessados para dar sequência a um segundo tópico de abordagem: analisar a Política Nacional de Segurança Alimentar e Nutricional, com base nas políticas públicas de Estado em nível nacional e principalmente alicerçado nos estudos de Recine e Vasconcellos (2011), Camilo et al. (2011) e Alves e Jaime (2014).

Dando continuidade aos procedimentos adotados, e com base nas duas etapas anteriores, buscou-se reavaliar as principais perspectivas existentes sobre o conceito de território para discuti-lo e aplicá-lo à

Hygeia Uberlândia - MG Edição especial: X GeoSaude $\quad$ Fev./2022 $\quad$ p. 34-45 Página 35


lógica de território alimentar urbano em disputa, no âmbito da Geografia da Saúde. Bases miltonianas como a de Saquet e Silva (2008) e Abrão (2010) auxiliaram nesse processo, bem como elementos presentes ao longo da investigação. Por fim, cabe ressaltar que os resultados aqui apresentados fazem parte de uma pesquisa mais ampla que está sendo desenvolvida na Universidade Federal do Paraná, que intenciona a médio prazo analisar em maiores detalhes a lógica espacial e os efeitos dos ultraprocessados no Paraná e no Brasil.

\section{A CADEIA ALIMENTAR ULTRAPROCESSADA}

Os ultraprocessados são produzidos para saciar os desejos alimentares, resultando em produtos considerados como hiperpalatáveis, ou seja, em um tipo de alimento que, a partir de suas características físico-químicas, estimulam e/ou alteram os mecanismos cerebrais que controlam o apetite, resultando desse processo um consumo excessivo destes alimentos, impedindo e/ou dificultando que as pessoas tenham um controle de suas dietas (MOUBARAC et al., 2015). Diante disso, o alimento do tipo ultraprocessado coloca-se como uma opção acessível pelas cadeias logísticas, além de ser apresentado como uma opção alimentar rápida e barata, a despeito de seu baixo valor nutritivo.

Em relação ao preço, a grande indústria alimentícia utiliza-se do avanço na produtividade e de um elevado investimento em pesquisa e desenvolvimento para produzir formulações alimentares cada vez mais baratas, além de um amplo suporte governamental de subsídios para as commodities, que participam e são essenciais na cadeia dos ultraprocessados (MONTEIRO, 2010). No Brasil, as indústrias também se valem do fato que o mercado de ultraprocessados possui grande potencial de crescimento, em grande medida por alguns drivers sócio-econômicos que são verificados no fenômeno latino-americano dessa cadeia, como por exemplo o processo de urbanização, elevação das rendas nos países da região, marketing, desregulamentação do mercado e mudanças na cadeia de distribuição de alimentos (MOUBARAC, 2015). Por essa razão, uma das característica fundantes desse novo sistema agroalimentar, está no fato de que a alimentação é encarada como um ativo derivativo, ou seja, tornando-se um ativo especulativo financeiro, de modo que as implicações dessa nova dinâmica "passam a incorporar variáveis emanadas das transações globais, de acordo com o grau de abertura das economias" (DE PAULA et al., 2015, p. 305).

Seguindo este raciocínio, Caivano (2017, p. 351) verifica que as "políticas econômicas que favorecem o fluxo do capital internacional, a entrada transnacional em mercados internos e a desregulamentação do mercado desde 1980" foram um dos principais motivadores para a constituição de grandes conglomerados alimentares que atuam na produção e distribuição da cadeia do ultraprocessado. Em vista disso, um relatório recente sobre ultraprocessados, elaborado pela Organização Mundial de Saúde (OMS) e pela Organização Pan-Americana da Saúde (PAHO), verificou com base em dados de 74 países que quando um governo desregulamenta o mercado alimentar, há uma tendência de crescimento da venda de ultraprocessados (MOUBARAC, 2015), reforçando o elo com as questões políticas e econômicas evidenciadas neste processo.

Após a década de 1990, em um contexto de desregulamentação e estabilização econômica, as grandes cadeias transnacionais e nacionais de supermercados intensificaram sua concentração de mercado sobre a cadeia de distribuição alimentar, totalizando portanto mais de 89 aquisições e fusões no período de 1995 a 2007 (MONTEIRO, 2012). De Oliveira (2015) pondera, entretanto, que no Brasil os 5 maiores grupos varejistas entre 1998 a 2013 controlaram aproximadamente $60 \%$ de toda a participação varejista, em grande parte devido a entrada de grandes grupos internacionais como, por exemplo, Carrefour e Walmart, que adquiriram uma série de redes nacionais nesse período e contribuíram para este processo de aglutinação de empresas e concentração no ramo.

Portanto, a questão levantada por Monteiro (2012) e De Oliveira (2015), a respeito da concentração de mercado no varejo alimentar, está centrada no controle das grandes redes globais de varejo sobre a mudança dos hábitos de consumo globais, onde pode-se arguir que esses grupos a partir de suas

$\begin{array}{lllll}\text { Hygeia Uberlândia - MG } \quad \text { Edição especial: X GeoSaude } \quad \text { Fev./2022 } & \text { p. 34-45 Página } 36\end{array}$


estratégias de poder estão colaborando majoritariamente em alterar os padrões alimentares e, com isso, inserir cada vez mais alimentos ultraprocessados para os consumidores, em especial aos de países subdesenvolvidos, cuja maior parte da população não possui necessariamente recursos financeiros para optar por comidas mais saudáveis no seu dia a dia.

Para além das redes de varejo, verifica-se que a grande indústria alimentícia brasileira se caracteriza pelo seu poder em estabelecer um amplo controle sobre diversos estágios da cadeia produtiva, incluindo em grande medida uma influência sobre o preço pago aos produtores, bem como a definição das estratégias de distribuição e promoção desses produtos (RAUD, 2008). Diante disso, é possível notar que nas últimas décadas houve crescimento significativo do consumo de alimentos ultraprocessados no Brasil, em especial devido aos preços desses alimentos estarem significativamente mais baratos ao longo dos anos em comparação com a alimentação tradicional. Em um cenário mais pessimista, Maia et al. (2020) imaginam que essas diferenças de preço entre os alimentos ultraprocessados e saudáveis devem continuar se acentuando, principalmente a partir do ano de 2030, onde os ultraprocessados devem se tornar ainda mais baratos que as comidas mais saudáveis.

Dessa forma, os estímulos observados nas últimas décadas na cadeira alimentar ultraprocessada seguramente contribuíram para uma maior participação destes mantimentos na dieta alimentar. Somado a esses fatores, as mudanças de hábitos na sociedade também favoreceram tal processo. Andrade (2007), por exemplo, verificou que o aumento das refeições fora de casa está positivamente associado com o consumo de alimentos ultraprocessados. Já em valores per capita, estima-se que, no ano 2000 , um cidadão brasileiro consumiu cerca de $86 \mathrm{~kg}$ de bebidas e alimentos ultraprocessados, enquanto no ano de 2013 esses valores aumentaram para 112,3 $\mathrm{kg}$, demonstrando uma taxa de crescimento de $2,1 \%$ ao ano (CAIVANO et al., 2017).

\section{OS ULTRAPROCESSADOS E SUAS CADEIAS DE DISTRIBUIÇÃO: A FORMAÇÃO DE DESERTOS E PÂNTANOS ALIMENTARES}

Um dos conceitos chave para compreender o impacto da grande indústria e a inserção de alimentos ultraprocessados no cotidiano da população está na terminologia de desertos e pântanos alimentares. Nesse sentido, deserto alimentar refere-se a "locais onde o acesso a alimentos in natura ou minimamente processados é escasso ou impossível" (IDEC, 2020, p. 1). Já os pântanos alimentares, em contrapartida, "são locais em que se predomina a venda de produtos altamente calóricos com poucos nutrientes, como no caso das redes de fast food e lojas de conveniência. Os dois cenários obrigam as pessoas a se locomover para outras regiões para obter alimentos saudáveis" (IDEC, 2020, p. 1).

Vandevijvere et al. (2019) também coloca o pântano alimentar como uma área em que há uma predominância de estabelecimentos que fornecem produtos ultraprocessados em comparação a oferta de alimentos saudáveis. Essencialmente, os dois conceitos se assemelham e são correlatos, entretanto cada um tem um intuito específico de análise, enquanto o deserto alimentar é centrado em definir áreas em que é difícil o acesso a alimentos saudáveis, o pântano alimentar refere-se a uma delimitação de regiões com elevada concentração de estabelecimentos com ultraprocessados. Nota-se que os dois conceitos podem ser utilizados para diferentes fins de planejamento territorial em saúde pública, entretanto torna-se importante a utilização de ambos, bem como o seu entendimento conceitual.

Honório (2020), em sua dissertação de mestrado sobre pântanos e desertos alimentares, pondera que há uma série de critérios ao redor do mundo para a delimitação destas áreas, entretanto nem sempre elas se apresentam tão adequadas para a realidade brasileira, pois no Brasil os supermercados são caracterizados como áreas de aquisição mista, ou seja, há a presença de alimentos não saudáveis e ultraprocessados no mesmo ambiente. Em algumas metodologias utilizadas no exterior, os mercados são considerados estabelecimentos de alimentação saudável. Tal descompasso frente à realidade brasileira resultou na necessidade da produção de um relatório técnico-metodológico denominado

\begin{tabular}{lllll}
\hline Hygeia & Uberlândia - MG & Edição especial: X GeoSaude & Fev./2022 & p. 34-45 Página 37
\end{tabular}


"Mapeamento dos Desertos Alimentares no Brasil", realizado pela Câmara Interministerial de Segurança Alimentar e Nutricional (CAISAN) onde foi "considerado como deserto alimentar os setores censitários que estavam abaixo do percentil 25 da densidade de estabelecimentos saudáveis" (HONORIO, 2020, p. 53). A metodologia da CAISAN (2018) pondera que os supermercados são estabelecimentos mistos, sendo também comercializados alimentos ultraprocessados, além das variedades in natura.

O transporte dos alimentos é uma variável importante na constituição de desertos alimentares. Com exceção do deslocamento por caminhada a pé, todos os meios de transporte exigem algum nível de acesso financeiro, mesmo que pontual (SHAW, 2006). No caso de andar a pé, Shaw (2006) pondera que é uma opção utilizada pelas classes sociais menos abastadas, entretanto pela limitação de peso para transportar objetos pesados, é rotineiro que as pessoas optem por carregar produtos não essenciais, como produtos de limpeza/higiene e rações de animais de estimação, não sobrando capacidade de carga para carregar alimentos saudáveis. Diante disso, os alimentos ultraprocessados, que costumam ser mais leves, tendem a ser mais escolhidos.

A distribuição desses alimentos pelo território está diretamente relacionada às mudanças nos sistemas alimentares que estão correlacionadas com as cadeias de distribuição globais de alimentos, desta forma verifica-se cada vez mais uma substituição de pequenos estabelecimentos especializados, pelo modelo uniformizado de supermercados de grandes cadeias globais (MONTEIRO et al., 2018). Nesse aspecto, o marketing é outra ferramenta largamente utilizada pelas grandes indústrias, enfatizando sempre características como praticidade e menor preço destes alimentos. A ampla concentração de mercado é outra característica presente na cadeia alimentar dos grandes grupos industriais. Fato esse que, conforme relatório do ETC Group de 2019, apenas 4 grandes multinacionais (Nestlé, Pepsico, Anheuser-Busch Inbev e JBS) detém mais de $54,5 \%$ de todo o market-share global da produção de alimentos (SHAND e WETTER, 2019).

Desse modo, segundo Goméz e Riketts (2013), há duas cadeias de distribuição alimentar distintas, sendo a primeira considerada a cadeia alimentar tradicional, composta por agricultores de pequeno porte, vendedores independentes e de porta a porta, pequenos negócios de bairro e barracas de rua. Já a segunda cadeia, denominada cadeia alimentar moderna, é relacionada diretamente à expansão do varejo supermercadista contemporâneo, geralmente composta por distribuidores, agronegócio e grandes indústrias alimentícias nacionais e internacionais, sendo diretamente vinculada ao ultraprocessado e a constituição de desertos e pântanos alimentares pelo território.

\section{POLÍTICA NACIONAL DE SEGURANÇA ALIMENTAR E NUTRICIONAL}

O direito humano à alimentação adequada (DHAA) é um dos direitos fundamentais que estão assegurados em uma série de documentos e pactos internacionais, entre eles a declaração universal dos Direitos Humanos de 1948, o pacto dos Direitos Econômicos, Culturais e Sociais, as cartas constitucionais nacionais e uma série de documentos elaborados pela Organização das Nações Unidas para Agricultura e Alimentação (FAO) (GAMBÁ e MONTAL, 2009). Nesse contexto a terminologia direito humano à alimentação adequada ultrapassa a barreira biológica de necessidade calórica que um indivíduo precisa para se manter vivo, de modo que para Canesqui e Garcia (2005), a alimentação deve ser entendida num elo entre a dimensão biológica e social, relacionando-se aos hábitos alimentares tradicionais e a qualidade dos nutrientes.

Especificamente sobre o cenário brasileiro, verifica-se que alimentação e nutrição são temáticas relevantes dentro do processo de reforma sanitária do estado brasileiro, especialmente com a promulgação da lei que regulamentou o SUS, a Lei Orgânica da Saúde em 1990. A vigilância nutricional e orientação alimentar se tornaram, portanto, diretrizes permanentes dentro do conceito de segurança alimentar e nutricional brasileiro (JAIME, 2018).

Nesse contexto, o estado brasileiro se caracteriza em dispor de um Programa Nacional de Alimentação e Nutrição (PNAN), sancionado em 1999 e que em sua última atualização, em 2011, apresentou como

$\begin{array}{lllll}\text { Hygeia Uberlândia - MG } & \text { Edição especial: X GeoSaude } & \text { Fev./2022 } & \text { p. 34-45 } & \text { Página } 38\end{array}$


diretrizes aspectos como garantia da qualidade alimentar, vigilância nutricional e alimentar, além do cuidado aos agravos relacionados a alimentação e nutrição (ALVES e JAIME, 2014). Outro arcabouço institucional recente que possibilitou desenvolver os mecanismos de gestão e governança em vigilância nutricional e alimentar é a Política Nacional de Segurança Alimentar e Nutricional (PNSAN), sendo ela a expressão prática e operacional das diretrizes de segurança alimentar contidas na Lei Orgânica de Segurança Alimentar e Nutricional (LOSAN), de modo que o planejamento da política em questão é dado pelo Plano Nacional de Segurança Alimentar e Nutricional (PLANSAN) (ALVES e JAIME, 2014; FREITAS, 2014).

Nesse conjuntura, a LOSAN também instituiu um sistema de coordenação nacional de segurança alimentar, denominado de Sistema Nacional de Segurança Alimentar e Nutricional (SISAN), sendo descrito por Freitas $(2018$, p.18) como um "sistema público que reúne os diversos setores de governo para a coordenação das políticas que têm o objetivo comum de promover a segurança alimentar e nutricional e o acesso digno à alimentação a toda a população". Outra relevante ferramenta inserida dentro do sistema de saúde, é o Sistema de Vigilância Alimentar e Nutricional (SISVAN), correspondendo a "a um sistema de coleta, processamento e análise contínua dos dados de uma população, possibilitando diagnóstico atualizado da situação nutricional, suas tendências temporais e também os fatores de sua determinação" (CAMILO et al., 2011, p.225).

Os dados do SISVAN são coletados a partir de uma estratégia de vigilância alimentar e nutricional (VAN), tendo em seu âmbito a utilização de profissionais de atenção à saúde primária que, além de coletarem os dados para o SISVAN, também desempenham um papel fundamental de elo entre as políticas públicas desenhadas e a sua efetiva concretização, conforme enfatizado por BORTOLINI et al. (2020). Dessa forma, o SISVAN é, portanto, uma ferramenta que pode auxiliar a detectar perfis alimentares, incluindo monitoramento da:

\begin{abstract}
“(...) situação de saúde, alimentar e nutricional de maneira sistemática revelando desigualdades entre regiões geográficas, grupos populacionais e populações vulneráveis e permite subsidiar intervenções voltadas para a prevenção e o controle de distúrbios nutricionais e doenças associadas à alimentação e nutrição e para a promoção de práticas alimentares e estilos de vida saudáveis" (RECINE e VASCONCELLOS, 2011, p. 74).
\end{abstract}

Do ponto de vista de políticas públicas, há uma organização legislativa sobre a forma que o Estado brasileiro deve se comportar quanto a problemática do DHAA e consequentemente aos desertos alimentares. Cabe ao Estado colocar em prática seu próprio arcabouço teórico já estabelecido, entretanto a realidade material pode implicar em uma série de empecilhos para a colocação em prática dessas políticas públicas. O ambiente político é determinante na garantia dos direitos a uma alimentação de qualidade, de modo que "o cenário atual do Brasil aponta para a desconstrução das políticas públicas sociais e anuncia processos de instabilidade na garantia dos direitos sociais" (GUERRA et al., 2021, p. 1240). Portanto, o sistema econômico é um dos principais responsáveis por desigualdades alimentares, sendo que Christiansen (2016) coloca que a noção dos desertos alimentares está diretamente vinculado ao sistema econômico vigente e a política neoliberal. $\mathrm{O}$ resultado prático disso tudo é a alimentação, que é um direito básico, sendo destinada a reproduzir-se enquanto mercadoria, ao invés de ser destinada a garantir qualidade de vida a todos.

\title{
A PANDEMIA DE COVID-19 E A RESTRIÇÃo AO DIREITO HUMANO À ALIMENTAÇÃo SAUDÁVEL
}

Como exposto no tópico anterior, o direito humano à alimentação saudável apresenta escopo legal em uma série de legislações e políticas públicas, de modo que se trata fundamentalmente de uma política de estado (COSTA, 2020). Nesse contexto, é verificável portanto que o SUS deve estar articulado com as demais políticas sociais para a garantia dos direitos, de modo que é dever institucional de todos os governantes promover uma quadro político e econômico que garanta todo esse arcabouço institucional, que por consequência garanta o acesso alimentar à população.

\begin{tabular}{llllll}
\hline Hygeia Uberlândia - MG & Edição especial: X GeoSaude & Fev./2022 & p. 34-45 Página 39
\end{tabular}


Entretanto, a trajetória brasileira recente demonstra uma escalada contra a garantia destes direitos, fato esse que teve o seu ápice com a eleição do governo Jair Bolsonaro em 2018. A partir de 2019, com o início do novo governo, há o efetivo "aprofundamento das contrarreformas iniciadas no governo anterior, verificando-se a aceleração e a intensificação das políticas que contribuem com o desmonte do Estado brasileiro" (BRAVO e MENEZES, 2021, p. 144). E com a pandemia de Covid-19, o descaso do governo com a saúde e com as políticas de controle ao vírus apenas refletem o histórico do que já estava posto, especialmente pela precarização contínua das políticas públicas. Silva e Gomes (2020, p. 2) conceituam portanto que "a Covid-19 tem relevado a perversidade da gestão do Estado na garantia dos direitos e das necessidades humanas básicas nos últimos anos".

Correlatamente, o preço da alimentação tornou-se cada vez mais expressivo para a população brasileira, especialmente num cenário de degradação de renda e inflação acentuada. Conforme o relatório da Rede Brasileira de Pesquisa em Soberania e Segurança Alimentar e Nutricional (Rede PENSSAN), o ano de 2021, sob o contexto da pandemia de Covid-19, demonstrou que "do total de 211,7 milhões de brasileiros(as), 116,8 milhões conviviam com algum grau de Insegurança Alimentar e, destes, 43,4 milhões não tinham alimentos em quantidade suficiente e 19 milhões de brasileiros(as) enfrentavam a fome" (PENSSAN, 2021, p. 10). Portanto, o resultado do relatório evidenciou que a crise econômica brasileira vem revertendo os ganhos sociais e o advento da pandemia intensificou o processo de insegurança alimentar. Fato esse verificado pelas estatísticas apuradas pelo relatório, demonstrando que o país saltou de "10,3 milhões de pessoas em insegurança alimentar em 2018 para 19,1 milhões em 2020" (PENSSAN, 2021, p. 53). Desse modo, o Brasil regrediu 15 anos de políticas sociais de garantia ao direito humano à alimentação adequada, resultando a um retorno dos patamares de 2004, demonstrando assim uma queda brutal nos índices brasileiros de segurança alimentar.

Em relação ao consumo dos ultraprocessados, a partir da queda de renda do brasileiro e a tendência significativa de redução de preços destes produtos frente aos alimentos saudáveis, conforme aponta o estudo de Maia et al. (2020), é eminente que os produtos ultraprocessados se tornam muitas vezes a única opção alimentar para muitas famílias. Lima (2021), em seu estudo de revisão sistemática da literatura, apresenta que uma quantidade significativa dos estudos apontaram alterações negativas dos hábitos alimentares dos brasileiros. Tendo em vista todas essas variáveis, é perceptível que o consumo de alimentos pouco nutritivos torne-se uma opção forçada para parcela significativa da população, especialmente em áreas mais pobres. Consequentemente, essa dinâmica corrobora com o processo de formação de desertos e pântanos alimentares, acirrando a lógica de um território alimentar que está em disputa.

\section{TERRITÓRIO ALIMENTAR URBANO EM DISPUTA}

A lógica de território na Geografia pode ser entendida em um encadeamento de conceitos, onde "o espaço contém o território modelado, configurado; o território corresponde aos complexos naturais e às construções/obras feitas pelo homem: estradas, plantações, fábricas, casas, cidades" (SAQUET e SILVA, 2008, p. 17). Nessa linha de pensamento, Tonucci Filho (2013. p. 47) prossegue afirmando que "o território poderia ser concebido a partir da imbricação de múltiplas relações de poder, do poder mais material das relações político-econômicas ao poder mais simbólico das relações de ordem cultural". Isto posto, o território pode ser palco da proliferação do capital, através da lógica da divisão social do trabalho, e representa palco dos conflitos e contradições sociais, podendo ser denominado como o locus desses acontecimentos (ABRÃO, 2010; SAQUET e SILVA, 2008).

Tendo em vista que o território pode ser visto como um espaço delimitado e que apresenta relações de poder, é possível avaliar e analisar suas dinâmicas e o impacto ao se pensar em saúde pública. Por esse ângulo, Gondim e Monken (2017) apontam que o território percebido pelo Estado brasileiro é um relevante promotor de desequilíbrios na relação homem e ambiente, tendo como consequências doenças e mortes derivadas das patologias. Os desertos e pântanos alimentares podem ser encarados, portanto, a partir de uma lógica de território, sendo por conseguinte um palco permanente de embates pela soberania alimentar e consequentemente a garantia de disposição espacial justa de acesso às

Hygeia Uberlândia - MG Edição especial: X GeoSaude $\quad$ Fev./2022 $\quad$ p. 34-45 Página 40


políticas de saúde. Por essa razão a garantia de concretização do direito à saúde está atrelado às formas que os serviços de saúde se dispõem no território, resultando num quadro que os territórios podem ser a possibilidade de efetivação destes direitos ou atuando contrariamente (FARIA, 2013).

Portanto, ao se pensar na dinâmica territorial, conforme apresentada e descrita nos estudos de Faria (2013), é importante compreender os territórios alimentares de uma ótica da territorialização geográfica. Isto é, territorializar em saúde é um conceito em que "populações, pessoas, grupos, organizações e instituições se fixam em um espaço, em determinado tempo (espaço temporal), e no qual organizam e estabelecem relações sociais que possibilitam criar identidade, vínculo e pertencimento ao lugar" (GONDIM e MONKEN, 2017, p. 36).

A alimentação é um elemento de disputa do território, constituindo assim uma noção de território alimentar em disputa. Nesse cenário de confronto pela hegemonia alimentar, grandes grupos de capital monopolistas avançam sobre o território, alterando a dinâmica da alimentação tradicional ao implementar uma visão mercantilista e puramente econômica sob o tema (MARINS et al., 2014). Portanto compreender essa problemática a partir da vigilância em saúde, é uma forma de pautar o território e propor uma nova matriz alimentar, que garanta principalmente a soberania alimentar das populações. A vigilância em saúde inserida dentro da atenção à saúde primária, deve se pautar a partir do "binômio os conhecimentos e as ações inerentes à vigilância epidemiológica, completando dessa forma a tríade: vigilância em saúde-vigilância epidemiológica-vigilância sanitária" (MARINS et al., 2014, p. 243).

\section{SUS COMO GARANTIA DO DIREITO À ALIMENTAÇÃO ADEQUADA}

Dentro do contexto brasileiro de inúmeras desigualdades sociais e espaciais, o SUS se apresenta como uma política pública norteadora para a garantia do direito humano à alimentação adequada. No entanto, para que essa característica se concretize, é fundamental a atenção à saúde primária seja caracterizada "pela longitudinalidade e integralidade nas ações e pela coordenação do cuidado a partir de uma perspectiva de integração dos serviços de saúde em determinado território" (BORTOLINI, 2020, p. 2). Ou seja, é importante que o território seja pautado pelo reconhecimento e análise, especificamente quanto aos critérios de riscos e vulnerabilidades.

A vigilância alimentar e nutricional é portanto um processo contínuo de avaliação e predição de "tendências das condições de alimentação e nutrição da população e de seus fatores determinantes para subsidiar o planejamento de ações para prevenção e enfrentamento dos agravos relacionados à alimentação e nutrição" (BORTOLINI, 2020, p. 2) Os pântanos e desertos ambientais são nesse contexto um exemplo de espacialização que nos ajuda a atender a distribuição do consumo de alimentos pelo território. Ao se utilizar estratégias territoriais a partir da saúde primária, o estado pode desenvolver uma política alimentar adequada a cada área específica.

Entre as ferramentas informacionais da qual o estado dispõe dentro da vigilância nutricional e alimentar, estão os registros do Sistema de Vigilância Alimentar e Nutricional (SISVAN), caracterizando-se por ser uma fonte de dados valiosa para a elaboração de políticas públicas de atenção à saúde primária. Bortolini (2020) pondera que a partir dos dados do SISVAN, as equipes de saúde primária podem desenvolver algumas estratégias junto à comunidade, vinculadas à promoção da alimentação adequada a partir das informações contidas no Guia Alimentar para a População Brasileira e Guia Alimentar para Crianças Brasileiras Menores de Dois Anos, orientando especialmente sobre os efeitos deletérios do consumo de alimentos ultraprocessados.

Para além disso, a proposição de hortas urbanas é uma outra opção que pode ampliar o acesso às comunidades a alimentação de qualidade, reduzindo os pântanos e desertos alimentares. A respeito desta proposta, espera-se estimular o contato da população com a produção de alimentos, pois tal fator "propicia conhecimentos e contribui para a formação de vínculo com o alimento produzido através da sensibilização para 0 ato de se alimentar, provocando mudanças não só nos que delas participam, mas também nas famílias e comunidades dos participantes" (GARCIA, 2016, p. 73).

\begin{tabular}{lllll}
\hline Hygeia Uberlândia - MG $\quad$ Edição especial: X GeoSaude & Fev./2022 & p. 34-45 & Página 41
\end{tabular}


Assim, o Sistema Único de Saúde (SUS), com a sua base organizacional territorial, deve ser um fator de enfrentamento dos desertos e pântanos a partir da lógica da atenção nutricional. A garantia de direitos básicos vincula-se à seus preceitos básicos, e na defesa de processos relacionados a "alimentação e nutrição voltados à promoção e proteção da saúde, prevenção, diagnóstico e tratamento de agravos, que devem estar associados às demais ações de atenção à saúde do SUS, para indivíduos" (BRASIL, 2012, p. 68).

\section{CONSIDERAÇÕES FINAIS}

O dilema do direito humano à alimentação adequada está pautado diretamente na condição contemporânea de disponibilidade alimentar que, por consequência, está alicerçada nas dinâmicas econômicas da grande indústria e suas práticas de fornecimento alimentar de ultraprocessados espalhadas pelo território. Nessa sistemática, pode-se afirmar que há, portanto, um embate de poder sobre o território, que nesse artigo denomina-se de território alimentar. Tal ambiente é composto por um emaranhado de atores em disputa, desde aos grandes grupos alimentares, aos grupos de resistência vinculados a formas de produção alimentar mais artesanais. Ambos os agentes participam e comercializam no território alimentar, entretanto, os grandes grupos globais, a partir de sua influência e poder, geralmente se sobrepõem sobre os demais e impõem sua lógica alimentar.

Em termos práticos, esse território alimentar em disputa promove espaços urbanos como os desertos e pântanos alimentares, sendo esses, a expressão da disputa alimentar pelo território urbano e consequentemente uma demonstração da influência que esses grupos econômicos conseguem perpetuar suas práticas alimentares. Uma possível solução, encontrada nesta investigação, é enfrentar o problema a partir de uma ótica territorial com as ferramentas que o nosso Sistema Único de Saúde já oferece.

A vigilância em saúde, pautada pela atenção à saúde primária a partir da lógica da atenção nutricional, é uma forma de combater a constituição desses desertos e pântanos, ao ponto que cabe então exigir politicamente para que seja colocado em prática todo o arcabouço teórico legislativo que já dispomos sobre alimentação saudável. É inquestionável que tivemos avanços significativos nos últimos anos no se refere a nossa legislação sobre a temática, porém, para os próximos anos, tal questão possivelmente será árdua e ainda mais desafiadora, tendo em vista as repercussões político-econômicas e as eventuais consequências e desdobramentos da pandemia de Covid-19 no Brasil e no Mundo. Especialmente num cenário de ampliação da fome e pobreza em nosso país, os dilemas e dificuldades tornam-se ainda mais complexos.

Nesse contexto, é ainda mais fundamental que seja realizada essa discussão sobre o direito humano à alimentação saudável, principalmente quando concebemos que a alimentação é muito além de suprir o organismo caloricamente e sim ato que permite a reprodução da vida de forma saudável, além de carregar consigo uma carga cultural e simbólica. Portanto superar os desertos e pântanos alimentares está também na percepção de fundar um novo território alimentar, que seja fundamentalmente democrático e respeitoso com as necessidades dos seres humanos.

\section{REFERÊNCIAS}

ABRÃO, Joice Aparecida Antonello. Concepções de espaço geográfico e território. Sociedade e Território, p. 46-64, 2010.

ALVES, Kelly Poliany de Souza; JAIME, Patricia Constante. A Política Nacional de Alimentação e Nutrição e seu diálogo com a Política Nacional de Segurança Alimentar e Nutricional. Ciência \&

Saúde Coletiva, v. 19, p. 4331-4340, 2014. https://doi.org/10.1590/1413-812320141911.08072014

ANDRADE, Giovanna Calixto. Consumo de alimentos ultraprocessados fora de domicílio no

Brasil (Tese de Doutorado). São Paulo: Universidade de São Paulo, 2007,

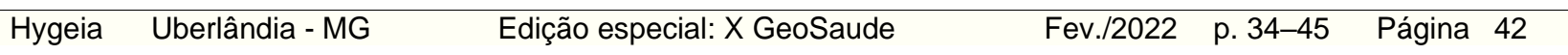


BORTOLINI, Gisele Ane et al. Ações de alimentação e nutrição na atenção primária à saúde no Brasil. Revista Panamericana de Salud Pública, v. 44, p. e39, 2020. https://doi.org/10.26633/RPSP.2020.39

BRASIL, Ministério da Saúde. Guia Alimentar para a População Brasileira. 2ª edição. Brasília: Ministério da Saúde, 2014.

BRASIL, Ministério da Saúde (MS). Secretaria de Atenção à Saúde. Departamento de Atenção Básica. Política Nacional de Alimentação e Nutrição Brasília: MS, 2012.

BRAVO, Maria Inês Souza; DE MENEZES, Juliana Souza Bravo. Saúde na atual conjuntura, a pandemia de coronavírus e as lutas da frente nacional contra a privatização da saúde. Humanidades \& Inovação, v. 8, n. 35, p. 143-156, 2021.

CAIVANO, Simone et al. Conflitos de interesses nas estratégias da indústria alimentícia para aumento do consumo de alimentos ultraprocessados e os efeitos sobre a saúde da população brasileira.

DEMETRA: Alimentação, Nutrição \& Saúde, v. 12, n. 2, p. 349-360, 2017. https://doi.org/10.12957/demetra.2017.26928

CAISAN. Estudo Técnico Mapeamento dos Desertos Alimentares no Brasil. Brasília: SecretariaExecutiva da Câmara Interministerial de Segurança Alimentar e Nutricional do Ministério do Desenvolvimento Social, 2018.

CAMILO, Stela Maria Bittencourt et al. Vigilância nutricional no Brasil: criação e implementação do SISVAN. Revista de APS, v. 14, n. 2, 2011.

CANESQUI, Ana María; GARCIA, Rosa Wanda Diez. Antropologia e nutrição: um diálogo possível. Rio de Janeiro: Editora Fiocruz, 2005.

COSTA, Fernanda Ferreira et al. Pandemia da Covid-19: Impactos à Renda e ao Aumento do Consumo de Alimentos Ultraprocessados. Revista Unimontes Científica, v. 22, n. 2, p. 1-15, 2020. https://doi.org/10.46551/ruc.v22n2a04

CHRISTIANSEN, Karina Hammershaimb. Reframing "Food Deserts": the history of urban supermarket access and its public policy discourse (Tese de Doutorado). Baltimore, Estados Unidos: Johns Hopkins University, 2016.

DE OLIVEIRA, Júlio César; DOS SANTOS DELGADO, Felipe. Análise da evolução da concentração industrial no varejo supermercadista brasileiro entre 1998 e 2013. Estudos do CEPE, n. 42, p. 41-60, 2015. https://doi.org/10.17058/cepe.v0i42.6207

DE PAULA, Nilson Maciel; SANTOS, Valéria Faria; PEREIRA, Wellington Silva. A financeirização das commodities agrícolas e o sistema agroalimentar. Estudos Sociedade e Agricultura, 2015.

FARIA Rivaldo Mauro. A territorialização da atenção primária à saúde no Sistema Único de Saúde e a construção de uma perspectiva de adequação dos serviços aos perfis do território. Hygeia, v. 9, n. 16, p. 121-130, 2013.

FREITAS, João Augusto de. O Sistema de Segurança Alimentar e Nutricional-SISAN: o desafio da intersetorialidade e da participação social na construção de uma política pública (Trabalho de Conclusão de Curso). São Paulo: Fundação Escola de Sociologia e Política de São Paulo, 2014.

GAMBÁ, Juliane Caravieri Martins; MONTAL, Zélia Maria Cardoso. Direito humano à alimentação adequada e responsabilidade internacional. Semina: Ciências Sociais e Humanas, v. 30, n. 1, p. 53-70, 2009.

GARCIA, Mariana Tarricone. Hortas urbanas e a construção de ambientes promotores da alimentação adequada e saudável. 2016. Tese de Doutorado. Universidade de São Paulo.

\begin{tabular}{lllll}
\hline Hygeia Uberlândia - MG $\quad$ Edição especial: X GeoSaude & Fev./2022 & p. 34-45 Página 43
\end{tabular}


GÓMEZ, Miguel I.; RICKETTS, Katie D. Food value chain transformations in developing countries: Selected hypotheses on nutritional implications. Food Policy, v. 42, p. 139-150, 2013. https://doi.org/10.1016/j.foodpol.2013.06.010

GONDIM, Grácia Maria de Miranda; MONKEN, Maurício. Território e Territorialização. Contexto e Identidade, p. 21, 2017.

GUERRA, Lúcia Dias da Silva; BEZERRA, Aída Couto Dinucci; CARNUT, Leonardo. Da fome à palatabilidade estéril: 'espessando' ou 'diluindo' o Direito Humano à Alimentação Adequada no Brasil?. Saúde em Debate, v. 44, p. 1231-1245, 2021. https://doi.org/10.1590/01031104202012721

HONÓRIO, Olivia Souza. Desertos e pântanos alimentares em uma metrópole brasileira (Dissertação de Mestrado). Belo Horizonte: Universidade Federal de Minas Gerais, 2020.

IDEC. Instituto Brasileiro de Defesa do Consumidor. Entre desertos e pântanos: quando a geografia urbana é um obstáculo para a alimentação saudável. 2020.

JAIME, Patricia Constante et al. Um olhar sobre a agenda de alimentação e nutrição nos trinta anos do Sistema Único de Saúde. Ciência \& Saúde Coletiva, v. 23, p. 1829-1836, 2018. https://doi.org/10.1590/1413-81232018236.05392018

MAIA, Emanuella Gomes et al. What to expect from the price of healthy and unhealthy foods over time? The case from Brazil. Public health nutrition, v. 23, n. 4, p. 579-588, 2020. https://doi.org/10.1017/S1368980019003586

LIMA, Evely Rocha et al. Implicações da pandemia de COVID-19 nos hábitos alimentares de brasileiros: revisão integrativa. Research, Society and Development, v. 10, n. 4, p. e29810414125e29810414125, 2021. https://doi.org/10.33448/rsd-v10i4.14125

MARINS, Bianca Ramos et al. Segurança alimentar no contexto da vigilância sanitária: reflexões e práticas. Rio de Janeiro: EPSJV/FIOCRUZ, 2014.

MARTINS, Ana Paula Bortoletto et al. Participação crescente de produtos ultraprocessados na dieta brasileira (1987-2009). Revista de Saúde Pública, v. 47, p. 656-665, 2013.

https://doi.org/10.1590/S0034-8910.2013047004968

MONTEIRO, Carlos Augusto et al. Increasing consumption of ultra-processed foods and likely impact on human health: evidence from Brazil. Public Health Nutrition, v. 14, n. 1, p. 5-13, 2010. https://doi.org/10.1017/S1368980010003241

MONTEIRO, Guilherme; FARINA, Elizabeth; NUNES, Rubens. Food-Retail Development and the Myth of Everyday Low Prices: The Case of Brazil. Development Policy Review, v. 30, n. 1, p. 49-66, 2012. https://doi.org/10.1111/j.1467-7679.2012.00559.x

MONTEIRO, Carlos Augusto et al. The UN Decade of Nutrition, the NOVA food classification and the trouble with ultra-processing. Public Health Nutrition, v. 21, n. 1, p. 5-17, 2018. https://doi.org/10.1017/S1368980017000234

MOUBARAC, Jean-Claude et al. Ultra-processed food and drink products in Latin America: Trends, impact on obesity, policy implications. Pan American Health Organization World Health Organization: Washington, DC, USA, p. 1-58, 2015.

PENSSAN, Rede. VIGISAN Inquérito Nacional sobre Insegurança Alimentar no Contexto da Pandemia da Covid-19 no Brasil. Rio de Janeiro: Rede Penssan. Disponível em: http://olheparaafome.com.br/VIGISAN Inseguranca alimentar.pdf. Acesso em: 10 nov. 2021.

RAUD, Cécile. Os alimentos funcionais: a nova fronteira da indústria alimentar análise das estratégias da Danone e da Nestlé no mercado brasileiro de iogurtes. Revista de sociologia e política, v. 16, p. 85-100, 2008. https://doi.org/10.1590/S0104-44782008000200008

\begin{tabular}{lllll}
\hline Hygeia Uberlândia - MG $\quad$ Edição especial: X GeoSaude & Fev./2022 & p. 34-45 Página 44
\end{tabular}


RECINE, Elisabetta; VASCONCELLOS, Ana Beatriz. Políticas nacionais e o campo da Alimentação e Nutrição em Saúde Coletiva: cenário atual. Ciência \& Saúde Coletiva, v. 16, n. 1, p. 73-79, 2011. https://doi.org/10.1590/S1413-81232011000100011

SAQUET, Marcos Aurelio; SILVA, Sueli Santos da. Milton Santos: concepções de geografia, espaço e território. GeoUERJ, v. 2, n. 18, p. 24-42, 2008.

SCRINIS, Gyorgy. Reformulation, fortification and functionalization: Big Food corporations' nutritional engineering and marketing strategies. The Journal of Peasant Studies, v. 43, n. 1, p. 17-37, 2016. https://doi.org/10.1080/03066150.2015.1101455

SHAND, Hope; WETTER, Kathy Jo (Org.). Mapping Corporate Power in Big Food. 2019.

SHAW, Hillary J. Food deserts: towards the development of a classification. Geografiska Annaler:

Series B, Human Geography, v. 88, n. 2, p. 231-247, 2006. https://doi.org/10.1111/j.04353684.2006.00217.x

VANDEVIJVERE, Stefanie et al. The first INFORMAS national food environments and policies survey in New Zealand: A blueprint country profile for measuring progress on creating healthy food environments. Obesity Reviews, v. 20, p. 141-160, 2019. https://doi.org/10.1111/obr.12850

TONUCCI FILHO, João Bosco Moura. Espaço e território: um debate em torno de conceitos-chave para a geografia crítica. Revista Espinhaço, p. 41-51, 2013. 